Bull. Austral. Math. Soc.

$26 \mathrm{~A} 18,54 \mathrm{H} 20,58 \mathrm{~F} 13$

VOL. 39 (1989) [259-264]

\title{
EVERY CHAOTIC INTERVAL MAP HAS A SCRAMBLED SET IN THE RECURRENT SET
}

\author{
BAU-SEN DU
}

Let $I$ denote a compact real interval and let $f \in C^{0}(I, I)$. In this note we show that if $f$ is chaotic in the sense of $\mathrm{Li}$ and Yorke, then there is an uncountable perfect $\delta$-scrambled set $S$ for $f$ in the recurrent set of $f$. Furthermore, the $w$-limit set of every $x \in S$ under $f$ contains $S$ and contains infinitely many periodic points of $f$ with arbitrarily large periods.

\section{INTRODUCTION}

Let $I$ denote a compact interval on the real line and let $f$ be a continuous map from $I$ into itself. For every positive integer $n$, let $f^{n}$ denote the $n$th iterate of $f: f^{1}=f$ and $f^{n}=f \circ f^{n-1}$ for $n>1$. Let $x_{0}$ be a point of $I$. $x_{0}$ is called a periodic point of $f$ if $f^{m}\left(x_{0}\right)=x_{0}$ for some positive integer $m$ and the smallest such positive integer $m$ is called the period of $x_{0}$ (under $f$ ). $x_{0}$ is called a recurrent point of $f$ if for every open neighbourhood $V$ of $x_{0}, f^{k}\left(x_{0}\right) \in V$ for some positive integer $k$. The set of all recurrent points of $f$ is called the recurrent set of $f$ and is denoted by $R(f)$. The set of all limit points of the set $\left\{x_{0}, f\left(x_{0}\right), f^{2}\left(x_{0}\right), \ldots\right\}=\left\{f^{n}\left(x_{0}\right) \mid n \geqq 0\right\}$ is called the $\omega$-limit set of $x_{0}$ under $f$ and is denoted by $\omega_{f}\left(x_{0}\right)$. If $f$ has a periodic point of period not an integral power of 2 , then we say that $f$ is chaotic in the sense of $\mathrm{Li}$ and Yorke $([3,6,8,11,16])$. Note that this is slightly different from the definition used in $[14,15]$ and $[18]$. It is well-known $([2,4,5,8-15,18,19])$ that if $f$ is chaotic in the sense of $\mathrm{Li}$ and Yorke, then there exist a positive real number $\delta$ and an uncountable perfect set $S$ (called a $\delta$-scrambled set of $f$ ) in $I$ such that the following hold:

(1) For any two distinct points $x$ and $y$ in $S$,

$$
\begin{gathered}
\liminf _{n \rightarrow \infty}\left|f^{n}(x)-f^{n}(y)\right|=0, \\
\limsup _{n \rightarrow \infty}\left|f^{n}(x)-f^{n}(y)\right| \geqq \delta .
\end{gathered}
$$

(2) For any point $x$ in $S$ and any periodic point $p$ of $f$,

$$
\limsup _{n \rightarrow \infty}\left|f^{n}(x)-f^{n}(p)\right| \geqq \delta / 2 \text {. }
$$

\section{Received 21 June 1988}

Copyright Clearance Centre, Inc. Serial-fee code: $0004-9729 / 89 \quad \$ A 2.00+0.00$. 
In this note we extend the ideas of Auslander and Yorke [1], and Osikawa and Oono [11] to show that if $f$ is chaotic in the sense of $\mathrm{Li}$ and Yorke, then the $\delta$-scrambled set $S$ mentioned above can be chosen in the recurrent set $R(f)$ of $f$. To be more precise, we prove the following result (see also [11]).

ThEOREM. If $f \in C^{0}(I, I)$ is chaotic in the sense of $L i$ and Yorke, then there exist a positive real number $\delta$ and an uncountable perfect set $S$ (called a $\delta$-scrambled set of $f$ ) in $R(f)$ such that the following hold:

(i) For every $x \in S$, the $\omega$-limit set of $x$ under $f$ contains $S$ and contains infinitely many periodic points of $f$ with arbitrarily large periods (see also [17]).

(ii) For any two distinct points $x$ and $y$ in $S$,

$$
\begin{array}{r}
\liminf _{n \rightarrow \infty}\left|f^{n}(x)-f^{n}(y)\right|=0, \\
\limsup _{n \rightarrow \infty}\left|f^{n}(x)-f^{n}(y)\right| \geqq \delta .
\end{array}
$$

(iii) For any point $x$ in $S$ and any periodic point $p$ of $f$,

$$
\limsup _{n \rightarrow \infty}\left|f^{n}(x)-f^{n}(p)\right| \geqq \delta / 2 .
$$

(iv) For any positive number $\varepsilon$, there are infinitely many periodic points $p$ of $f$ such that, for every $x \in S$,

$$
\liminf _{n \rightarrow \infty}\left|f^{n}(x)-p\right|<\varepsilon
$$

\section{PROOF OF THE THEOREM}

We first recall some terminology from symbolic dynamics. Let $\Sigma_{2}=\{a \mid a=$ $\left(a_{0}, a_{1}, a_{2}, \cdots\right), a_{k}=0$ or 1 for all $\left.k \geqq 0\right\}$. A metric for $\Sigma_{2}$ is given by putting $d\left(\left(a_{0}, a_{1}, a_{2}, \cdots\right),\left(b_{0}, b_{1}, b_{2}, \cdots\right)\right)=\Sigma\left|a_{k}-b_{k}\right| / 2^{k}$. Let $\sigma: \Sigma_{2} \rightarrow \Sigma_{2}$ be the shift map $\sigma\left(\left(a_{0}, a_{1}, a_{2}, \cdots\right)\right)=\left(a_{1}, a_{2}, \ldots\right)$, that is $(\sigma(a))_{k}=a_{k+1}$ for all $k \geqq 0$ if $a=\left(a_{0}, a_{1}, a_{2}, \cdots\right)$. Then $\left(\Sigma_{2}, d\right)$ is a compact metric space and $\sigma$ is a continuous, onto, two to one map. The pair $\left(\Sigma_{2}, \sigma\right)$ is called the one-sided shift map on two symbols. In the following, we omit parentheses and commas in writing the elements of $\Sigma_{\mathbf{2}}$. We call $\left[a_{0}\left|a_{1}\right| \cdots \mid a_{k-1}\right]=\left\{\omega \in \Sigma_{2} \mid \omega=\omega_{0} \omega_{1} \omega_{2} \cdots\right.$ and $\omega_{j}=a_{j}$ for $\left.0 \leqq j \leqq k-1\right\}$ a $k$-cylinder in $\Sigma_{2}$. We note that there are uncountably many elements $\omega \in \Sigma_{2}$ (called transitive elements) such that for every cylinder set $Q$ in $\Sigma_{2}, \sigma^{m} \omega \in Q$ for infinitely many positive integers $m$.

Let $g \in C^{0}(I, I)$. Assume that there are two disjoint closed subintervals $I_{0}, I_{1}$ of $I$ such that $g\left(I_{0}\right) \cap g\left(I_{1}\right) \supseteq I_{0} \cup I_{1}$. Let $Z=I_{0} \cup I_{1}$. For all $a_{k}=0$ or 1 , 
$k \geqq 0$, we define $I\left(a_{0} a_{1} \ldots a_{k}\right)$ inductively by putting $I(0)=I_{0}, I(1)=I_{1}$, and letting $I\left(a_{0} a_{1} \cdots a_{k+1}\right)$ be a closed subinterval of $I\left(a_{0} a_{1} \cdots a_{k}\right)$ such that $g\left(I\left(a_{0} a_{1} \cdots a_{k+1}\right)\right)=$ $I\left(a_{1} a_{2} \cdots a_{k+1}\right)$. For every $a=a_{0} a_{1} a_{2} \cdots \in \Sigma_{2}$, let $I(a)=\bigcap_{k} I\left(a_{0} a_{1} \cdots a_{k}\right)$. Then $I(a)$ is either a compact interval or consists of one point and $g(I(a))=I(\sigma a)$. Let $Z^{*}$ be the union of $I(a)$ for all $a \in \Sigma_{2}$ and let $B$ be the set of all $b \in \Sigma_{2}$ with $I(b)$ consisting of one point. It is easy to see that $Z^{*}$ is a nonempty compact subset of $Z$ with $g\left(Z^{*}\right)=Z^{*}$. Let $h: Z^{*} \rightarrow \Sigma_{2}$ be defined by putting $h(z)=a=a_{0} a_{1} a_{2} \cdots$ for $z \in Z^{*}$, where $a_{n}=k$ if $g^{n}(z) \in I_{k}, n \geqq 0$. Note that the map $h$ defined above is the same as the map $\tau: Z^{*} \rightarrow \Sigma_{2}$ defined by putting $\tau(z)=a$ for every $z \in I(a) \subset Z^{*}$. Now we can state the following result (see also [1]).

Lemma 1. Let $g, Z^{*}$, and $h$ be defined as above. Then the following hold.

(a) $h$ is continuous and onto.

(b) $g$ is semiconjugate through $h$ to $\sigma$ on $Z^{*}$, that is $h g=\sigma h$ on $Z^{*}$.

(c) If $z \in Z^{*}, h(z)=a$, and $\overline{\left\{g^{n}(z) \mid n \geqq 0\right\}}$ contains a periodic point of $g$ with period $m$, then $\overline{\left\{\sigma^{n}(a) \mid n \geqq 0\right\}}$ contains a periodic point of $\sigma$ with period dividing $m$. In particular, $h$ sends periodic points of $g$ in $Z^{*}$ into periodic points of $\sigma$.

(d) If $a \in \Sigma_{2}$ is a periodic point of $\sigma$ with period $m$, then $I(a)$ contains a periodic point of $g$ with period $m$. Furthermore, if $a \notin B$, then at least one endpoint of $I(a)$ is a periodic point of $g$ with period $m$ or $2 m$. Consequently, for every positive integer $m, g$ has at least as many periodic points of period $m$ as the shift map $\sigma$ in $\Sigma_{2}$.

(e) $h$ sends recurrent points of $g$ in $Z^{*}$ into recurrent points of $\sigma$.

(f) If $a \in \Sigma_{2}$ is a recurrent point of $\sigma$, then $I(a)$ contains a recurrent point of $g$. Furthermore, if $a \notin B$, then at least one endpoint of $I(a)$ is a recurrent point of $g$.

(g) Assume that $b \in \Sigma_{2}-B$ is not transitive for $\sigma$. Then there is an endpoint $y$ of $I(b)$ such that if $I\left(\sigma^{n} b\right)$ is a nondegenerate closed interval for some positive integer $n$, then $g^{n}(y)$ is an endpoint of $I\left(\sigma^{n} b\right)$.

(h) For every transitive element $b \in \Sigma_{2}$ of $\sigma$, the $\omega$-limit set of $x_{b}$ under $g$ for every $x_{b} \in I(b)$ contains all elements of $I(c)$ with $c \in B$ and contains at least one endpoint of $I(a)$ for every point $a \in \Sigma_{2}-B$. In particular, if $b \in \Sigma_{2}$ is transitive for $\sigma$, then the $\omega$-limit set $\omega_{g}\left(x_{b}\right)$ of $x_{b}$ under $g$ for every $x_{b} \in I(b)$ contains infinitely many periodic points of $g$. Consequently, there are uncountably many points in $Z^{*}$ which are not asymptotically periodic.

Proof: We give a proof of $(h)$ only. The other proofs are easy and are omitted. Let $a$ be a transitive element of $\sigma$ in $B$ with $I(a)=\left\{x_{a}\right\}$. Let $c \in B$ and $I(c)=\left\{x_{c}\right\}$. Then, for $\varepsilon>0$, there is a positive integer $m$ such that $I\left(c_{0} c_{1} \cdots c_{k}\right) \subseteq\left(x_{c}-\varepsilon, x_{c}+\varepsilon\right)$ for all $k \geqq m$. Since $a$ is transitive, $\sigma^{n} a \in\left[c_{0}\left|c_{1}\right| \cdots \mid c_{m}\right]$ for infinitely many positive integers $n$. Hence, $g^{n}\left(x_{a}\right) \in g^{n}(I(a))=I\left(\sigma^{n} a\right) \subset I\left(c_{0} c_{1} \cdots c_{m}\right) \subseteq\left(x_{c}-\varepsilon, x_{c}+\varepsilon\right)$ for 
infinitely many positive integers $n$. This shows that $x_{c} \in \omega_{g}\left(x_{a}\right)$.

Now let $c \notin B$ and $I(c)=[y, z]$ with $y<z$. Since $I(a)$ consists only of the point $x_{a}$ and $I(c)=[y, z]$ is a nondegenerate interval, we see that $I\left(\sigma^{n} a\right)=g^{n}(I(a)) \neq I(c)$ for all $n \geqq 0$. Hence, $\sigma^{n} a \neq c$ for all $n \geqq 0$. By a similar argument to that above, we see that, for any $\epsilon>0, g^{n}\left(x_{a}\right) \in g^{n}(I(a))=I\left(\sigma^{n} a\right) \subseteq(y-\varepsilon, z+\varepsilon)-[y, z]=$ $(y-\varepsilon, y) \cup(z, z+\varepsilon)$ for infinitely many positive integers $n$. Consequently, $y \in \omega_{g}\left(x_{a}\right)$ or $z \in \omega_{g}\left(x_{a}\right)$.

In the following, we use a method introduced in [11]. We first let the map $\phi$ from $\Sigma_{2}$ into $Z^{*}$ be defined as in $[11]$ by the following five steps:

Step 1 For $a$ such that $I(a)$ consists of a point $x_{a}$, let $\phi(a)=x_{a}$.

Step 2 If $\phi(a)$ is defined for $a$, let $\phi(\sigma(a))=g(\phi(a))$.

Step 3 If $\phi(\sigma(a))$ is defined for $a$, let $\phi(a) \in I(a)$ be defined such that $g(\phi(a))=$ $\phi(\sigma(a))$.

Step 4 If $\phi$ is not yet defined on $\sigma^{k}(a)$ for all integers $k \geqq 0$ and no $\sigma^{k}(a)$ is a periodic point of $\sigma$, let $\phi(a)$ be chosen as an arbitrary point in $I(a)$.

Step 5 If $\phi$ is not yet defined on $\sigma^{k}(a)$ for all integers $k \geqq 0$ and $a$ is a periodic point of $\sigma$ with period $n \geqq 1$, let $\phi(a)$ be chosen as an arbitrary point in $I(a)$ such that $g^{n}(\phi(a))=\phi(a)$ (such $\phi(a)$ exists since $g^{n}(I(a))=$ $\left.I\left(\sigma^{n}(a)\right)=I(a)\right)$.

Since, for every $a \in \Sigma_{2}$, we have $\phi(a) \in I(a)$, it is clear that $\phi$ is one-toone, and from the construction of $\phi, g \phi=\phi \sigma$ on $\Sigma_{2}$. Note that if $a \in \Sigma_{2}$ is a periodic point of $\sigma$, then $\phi(a)$ is a periodic point of $g$, and vice versa. From now on, we let $a=a_{0} a_{1} a_{2} \ldots \in \Sigma_{2}$ be any transitive point such that $I(a)$ consists of one point. For any point $b=b_{0} b_{1} b_{2} \cdots \in \Sigma_{2}$, define $\omega_{b} \in \Sigma_{2}$ by putting $\omega_{b}=a_{0} b_{0} a_{0} a_{1} b_{0} b_{1} a_{0} a_{1} a_{2} b_{0} b_{1} b_{2} \cdots a_{0} a_{1} a_{2} \cdots a_{k} b_{0} b_{1} b_{2} \cdots b_{k} \cdots$ and let $T=\left\{\phi\left(\omega_{b}\right) \mid\right.$ $b \in \Sigma_{2}$ and $I\left(\omega_{b}\right)$ consists of one point $\}$. Then $T$ is an uncountable Borel set. We now show that $g$ behaves chaotically on $T$.

Let $b=b_{0} b_{1} b_{2} \cdots \in \Sigma_{2}$ and $c=c_{0} c_{1} c_{2} \cdots \in \Sigma_{2}$ with $b \neq c$. Then there exists a least integer $k \geqq 0$ such that $b_{k} \neq c_{k}$. Since $a$ is transitive, $g^{m}\left(\phi\left(\omega_{b}\right)\right) \in I\left(b_{k}\right)$ and $g^{m}\left(\phi\left(\omega_{c}\right)\right) \in I\left(c_{k}\right)$ for infinitely many $m$. Therefore, $\underset{n \rightarrow \infty}{\limsup }\left|g^{n}\left(\phi\left(\omega_{b}\right)\right)-g^{n}\left(\phi\left(\omega_{c}\right)\right)\right| \geqq$ $\operatorname{dist}\left(I_{0}, I_{1}\right)$.

On the other hand, $\left\{\sigma^{n(n+1)}\left(\omega_{b}\right), \sigma^{n(n+1)}\left(\omega_{c}\right)\right\} \subseteq\left[a_{0}\left|a_{1}\right| \cdots \mid a_{n}\right]$ for all $n \geqq 1$ and $\phi\left(\left[a_{0}\left|a_{1}\right| \cdots \mid a_{n}\right]\right) \subseteq I\left(a_{0} a_{1} \cdots a_{n}\right)$ converges to the set $I(a)$ which consists of one point. So, $\liminf _{n \rightarrow \infty}\left|g^{n}\left(\phi\left(\omega_{b}\right)\right)-g^{n}\left(\phi\left(\omega_{c}\right)\right)\right|=0$.

Now let $p$ be any periodic point of $g$ with period $k$. Since $a$ is transitive, there are infinitely many integers $m$ and $n$ such that $a_{m}=a_{m+1}=\cdots=a_{m+k+1}=0$ and $a_{n}=$ $a_{n+1}=\cdots=a_{n+k+1}=1$. Consequently, $\limsup _{n \rightarrow \infty}\left|g^{n}\left(\phi\left(\omega_{b}\right)\right)-g^{n}(p)\right| \geqq \operatorname{dist}\left(I_{0}, I_{1}\right) / 2$. 
Finally, for any $\varepsilon>0$, since $I(a)=\{\phi(a)\}$, there is a cylinder $Q$ such that the length of the smallest interval $V$ containing $\phi(Q)$ is less than $\varepsilon$. Since $Q$ contains infinitely many periodic points of $\sigma, \phi(Q) \subseteq V$ contains infinitely many periodic points $p$ of $g$. Since $a$ is transitive, we see that, for every $x \in T, g^{n}(x) \in V$ for infinitely many $n \geqq 1$. Consequently, we have, for every $x \in T$,

$$
\liminf _{n \rightarrow \infty}\left|g^{n}(x)-p\right|<\varepsilon \text {. }
$$

It is well-known [7] that every uncountable Borel set contains a perfect set. Let $S$ denote any such perfect set in $T$. From what we have proved above, together with Lemma 1, we obtain immediately the following result.

Lemma 2. Let $g \in C^{0}(I, I)$. Assume that there exist two disjoint closed subintervals $I_{0}, I_{1}$ of $I$ such that $g\left(I_{0}\right) \cap g\left(I_{1}\right) \supseteq I_{0} \cup I_{1}$. Let $\delta=\operatorname{dist}\left(I_{0}, I_{1}\right)$. Then there exists an uncountable perfect set $S$ (called a $\delta$-scrambled set of $g$ ) in $R(g$ ) such that the following hold.

(i) For every $x \in S$, the $\omega$-limit set of $x$ under $g$ contains $S$ and contains infinitely many periodic points of $g$ with arbitrarily large periods.

(ii) For any two distinct points $x$ and $y$ in $S$,

$$
\begin{aligned}
& \liminf _{n \rightarrow \infty}\left|g^{n}(x)-g^{n}(y)\right|=0, \\
& \limsup _{n \rightarrow \infty}\left|g^{n}(x)-g^{n}(y)\right| \geqq \delta .
\end{aligned}
$$

(iii) For any point $x$ in $S$ and any periodic point $p$ of $g$,

$$
\underset{n \rightarrow \infty}{\limsup }\left|g^{n}(x)-g^{n}(p)\right| \geqq \delta / 2 \text {. }
$$

(iv) For any positive number $\varepsilon$, there are infinitely many periodic points $p$ of $g$ such that, for every $x \in S$,

$$
\liminf _{n \rightarrow \infty}\left|g^{n}(x)-p\right|<\varepsilon
$$

Now we can prove the theorem. Assume that $f$ has a periodic point of period $2^{m}(2 n+1)$. Then $f^{2^{m}}$ has a periodic point of period $2 n+1$. By Sharkovskii's theorem [16], the map $g=f^{2^{m+1}}$ has a periodic point of period 3 . Without loss of generality, we may assume that $x<y<z, g(x)=y, g(y)=z$ and $g(z)=x$. So, there is a point $w \in(y, z)$ such that $g(w)=y$. Let $I_{0}=[x, y]$ and $I_{1}=[w, z]$. Then it is easy to see that $g^{3}\left(I_{0}\right) \cap g^{3}\left(I_{1}\right) \supseteq I_{0} \cup I_{1}$. The theorem now follows easily from Lemma 2 since $R(f)=R(g)$. This completes the proof of the theorem. 


\section{REFERENCES}

[1] J. Auslauder and J.A. Yorke, 'Interval naps, factors of maps, and chaos', Tôhoku Math. J. 32 (1980), 177-188.

[2] A.M. Bruckner and Thakyin $\mathrm{Hu}$, 'On scrambled sets for chaotic functions', Trans. Amer. Math. Soc. 301 (1987), 289-297.

[3] G.J. Butler and G. Pianigiani, 'Periodic points and chaotic functions in the unit interval', Bull. Austral. Math. Soc. 18 (1978), 255-265.

[4] T. Gedeon, 'There are no chrotic mappings with residual scrambled sets', Bull. Austral. Math. Soc. 36 (1987), 411-416.

[5] I. Kan, 'A chaotic function possessing a scrambled set with positive Lebesgue measure', Proc. Amer. Math. Soc. 82 (1984), 45-49.

[6] P.E. Kloeden, 'Chaotic difference equations are dense', Bull. Austral. Math. Soc. 15 (1976), 371-379.

[7] K. Kuratowski, Topologie I (PWN - Polish Scientific Publishers, Warsaw, 1968). p. 387 .

[8] T.-Y. Li and J.A. Yorke, 'Period three implies chaos', Amer. Math. Monthly 82 (1975), 985-992.

[8] M. Misiurewicz, 'Chaos almost everywhere', Lect. Notes in Math. 1163. pp. 125-130 (SpringerVerlag).

[10] I. Mizera, 'Continuous chaotic functions of an interval have generically small scrambled sets', Bull. Austral. Math. Soc. 37 (1988), 89-92.

[11] M. Osikawa and Y. Oono, 'Chaos in $C^{0}$-endomorphism of interval', Publ. Res. Inst. Math. Sci. 17 (1981), 165-177.

[12] J. Smital, 'A chaotic function with some extremal properties', Proc. Amer. Math. Soc. 87 (1983), 54-56.

[13] J. Smital, 'A chaotic function with scrambled set of positive Lebesgue measure', Proc. Amer. Math. Soc. 82 (1984), 50-54.

[14] J. Smital, 'Chaotic functions with zero topological entropy', Thans. Amer. Math. Soc. 287 (1986), 269-282.

[15] J. Smital and K. Jankova, 'A characterization of chaos', Bull. Austral. Math. Soc. 34 (1986), 283-292.

[16] A.N. Sharkovskii, 'Coexistence of cycles of a continuous mapping of the real line into itselr, (Russian), Ukrain. Mat. Zh. 16 (1964), 61-71.

[17] A.N. Sharkovskii, 'Behavior of a mapping in the neighbourhood of an attracting set', (Russian), Ukrain. Mat. Zh. 18 (1966), 60-83. (English), AMS Trans. (2) 97 (1970), 227-258.

[18] J.-C. Xiong, 'A chaotic map with topological entropy 0', Acta Math. Sci. B (1986), 439-443.

[19] Z.-L. Zhou, 'A note on the Li-Yorke theoren', Kexue Tongbao (English Ed.) 31 (1986), 649-651.

Institute of Mathematics

Academia Sinica

Tajpei, Taiwan 11529

Republic of China 